\title{
ECTASIA QUÍSTICA DE LA RETE TESTIS.
}

\author{
Carlos Pascual Mateo, Inmaculada Fernández González', Marcos Luján Galán, Nuria \\ Rodríguez García, Gino Espinales Castro y Antonio Berenguer Sánchez.
}

Servicio de Urología. Hospital Universitario de Getafe. Madrid. y

Servicio de Urología'. Hospital de la Princesa. Madrid. España.

\begin{abstract}
Resumen.- OBJETIVO: La ectasia quística de la rete testis es una entidad benigna y típicamente en la ecografía aparece como una colección de pequeñas estructuras anecoicas en la confluencia del mediastinum testis. La importancia clínica de esta entidad radica en hacer un adecuado diagnóstico diferencial con la neoplasia testicular con componente quístico

MÉTODO: De forma retrospectiva, se revisa en la base de datos de la Unidad de Ecografía del Servicio de Urología el número de pacientes diagnosticados de ectasia quística de la rete testis durante un periodo de 6 años.
\end{abstract}

RESULTADOS: Durante los 6 años revisados se han diagnosticado 3 casos de ectasia quística de la rete testis y la ecografía fue realizada por dolor testicular. La edad media de los pacientes fue de 62 años. Ningún paciente ha desarrollado durante el tiempo de seguimiento un tumor testicular

CONCLUSIONES: El conocimiento de las características ecográficas encontradas en la ectasia quística de la rete testis permite hacer un adecuado diagnóstico de esta entidad benigna sin necesidad de recurrir a la biopsia testicular

Palabras clave: Ectasia quística. Rete testis.

Carlos Pascual Mateo

Servicio de Urología.

Hospital Universitario de Getafe.

Ctra de Toledo, Km. 12.500

28905 Getafe. Madrid. (España)

carlospascualmateo@yahoo.es

Trabajo recibido: 1 de abril 2005
Summary.- OBJECTIVES: The cystic ectasia of the rete testis is a benign entity with a typical ultrasound appearance as a collection of small hypoechoic structures in the confluence of the mediastinum testis. The clinical importance of these entity remains on doing an adequate differential diagnosis with testicular neoplasias with a cystic component.

METHODS: We retrospectively reviewed the database of the Ultrasound Unit in the Department of Urology looking for patients with a diagnosis of cystic ectasia of the rete testis over a six-year period.

RESULTS: Three cases of cystic ectasia of the rete testis were diagnosed over the six-year period, in all the indication for ultrasound was testicular pain. Mean patient age was 62 years. No patient developed testicular tumor on follow-up.

CONCLUSIONS: The knowledge of the ultrasound characteristics found in the cystic ectasia of the rete testis helps to make a proper diagnosis of this benign entity without the need of indication of testicular biopsy.

Keywords: Cystic ectasia. Rete testis

\section{INTRODUCCIÓN}

La ectasia quística de la rete testis es un hallazgo poco frecuente, que generalmente se diagnostica de forma incidental durante la realización de una ecografía escrotal que se indica por dolor o masa escrotal. Se trata de una entidad benigna y típicamente en la ecografía aparece como una colección de pequeñas estructuras anecoicas en la confluencia del mediastinum testis $(1,2)$. La importancia clínica de esta entidad radica en hacer un adecuado diagnóstico diferencial con la neoplasia testicular con componente quístico. 


\section{MATERIAL Y MÉTODO}

De forma retrospectiva, se revisa en la base de datos de la Unidad de Ecografía del Servicio de Urología el número de pacientes diagnosticados de ectasia quística de la rete testis durante un periodo de 6 años.

Para la realización de la ecografía escrotal el paciente se coloca en decúbito supino y se utiliza un transductor lineal de alta frecuencia. Se visualiza el testículo, en cortes transversales y longitudinales, el epidídimo, las estructuras paratesticulares y el cordón espermático. En todos los casos se hace una ecografía doppler color.

La apariencia ecográfica característica de la rete testis es la de una estructura ecogénica que se comunica desde el mediastinum testis con la cabeza epididimaria. Ocasionalmente puede ser anecoica con estructuras redondas o tubulares, numerosas y de pequeño tamaño que indican la existencia de una ectasia tubular de la rete testis. No existen criterios en la literatura en cuanto al número y tamaño de los quistes para diagnosticar una ectasia quística.

\section{RESULTADOS}

Durante los 6 años revisados se han diagnosticado 3 casos de ectasia quística de la rete testis y la ecografía fue realizada por dolor testicular. La edad media de los pacientes fue de 62 años. En cada caso se observaron estructuras quísticas a nivel de la rete testis sin componente sólido ni calcificación y sin flujo cuando se realizó el eco Doppler color. En 1 caso fue bilateral y en 2 unilateral. Un paciente tenía el antecedente de quistes epididimarios, otro paciente de epididimitis de repetición y en otro paciente no se encontraron antecedentes. Aunque se les ha diagnosticado de una patología benigna se ha realizado un seguimiento anual con exploración física y ecografía, sin hacer biopsia testicular y no han desarrollado un tumor testicular hasta la actualidad.

\section{DISCUSIÓN}

La rete testis está compuesta por 3 segmentos: la porción septal, formada por los túbulos rectos; la porción mediastínica, consistente en canales aplanados que discurren paralelos al hilio y la porción extratesticular, donde los canales se dilatan para formar pequeñas cavidades. Esta porción acaba en los ductos eferentes. Los túbulos seminíferos drenan a traves de aproximadamente 1400 comunicaciones en la porción septal de la rete testis (3).

La obliteración parcial o completa de los ductos eferentes origina una ectasia de la rete testis que eventualmente origina una ectasia quística de la rete testis. La obstrucción se puede originar en la unión testículo-epidídimo, en el interior del epidídimo o en la porción inicial de los ductos eferentes (4). Los mecanismos de obstrucción pueden ser debidos a diferentes causas. Los mecanismos más frecuente son: a) mecánico, por compresión de los ductos ex-
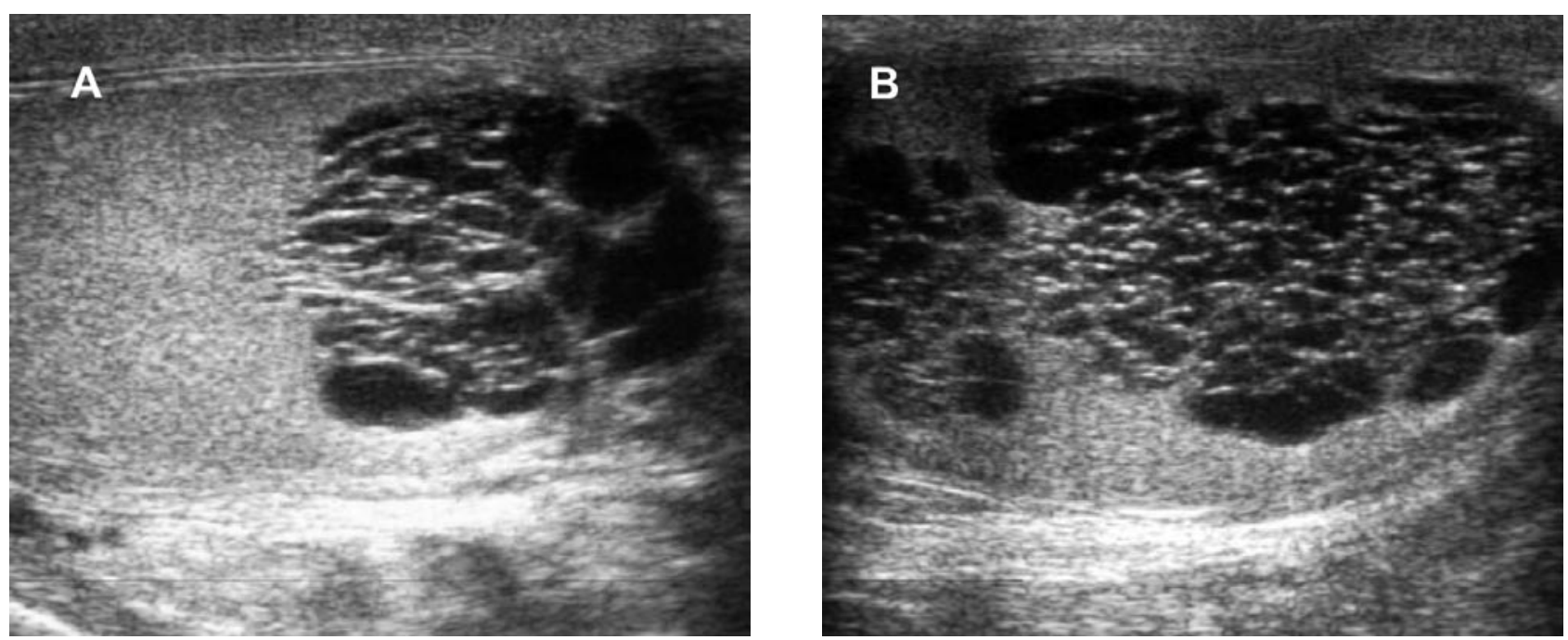

FIGURA 1. Se observan estructuras quísticas a nivel de la rete testis sin componente sólido ni calcificación que se corresponde con el diagnóstico ecográfico de ectasia quística de la rete testis.

A: Sección longitudinal. B: Sección transversal. 
cretores extratesticulares (tumor epididimario, tumores del cordón espermático que infiltran el epidídimo, epididimitis, traumatismos, cirugía, espermatocele y eventualmente la vasectomía); b) malformativo, por la disociación teste-epidídimo que puede observarse en la criptorquidia; c) isquémico por lesión arterial que origina una atrofia epididimaria; $y$ d) hormonal, por un disbalance andrógeno-estrogénico $(2,3)$.

Histológicamente Nistal y cols. (3) describen dos tipos de ectasia quística, denominadas con o sin metaplasia epitelial; la simple ectasia quística sin metaplasma epitelial se asocia generalmente con condiciones extratesticulares subyacentes que originan obstrucción de los ductos; la ectasia quística con metaplasma epitelial habitualmente se asocia con patología intratesticular, fundamentalmente tumor y criptorquidia.

La ectasia quística de la rete testis generalmente aparece en varones mayores de 55 años y es bilateral en el $29-69 \%$ de los casos y generalmente asimétrica $(2,5)$.

En la ecografía aparece como una colección de pequeñas estructuras anecoicas en la confluencia del mediastinum testis y con frecuencia se observan quistes epididimarios. Estas características ayudan a diferenciar la ectasia quística de la rete testis de tumores testiculares malignos con componente quístico. El teratoma es el que con más frecuencia se manifiesta con masas quísticas; no obstante los tumores quísticos son raros y cuando aparecen los quistes no cumplen los criterios de un quiste simples. Habitualmente el tumor quístico está rodeado de una "cáscara" de

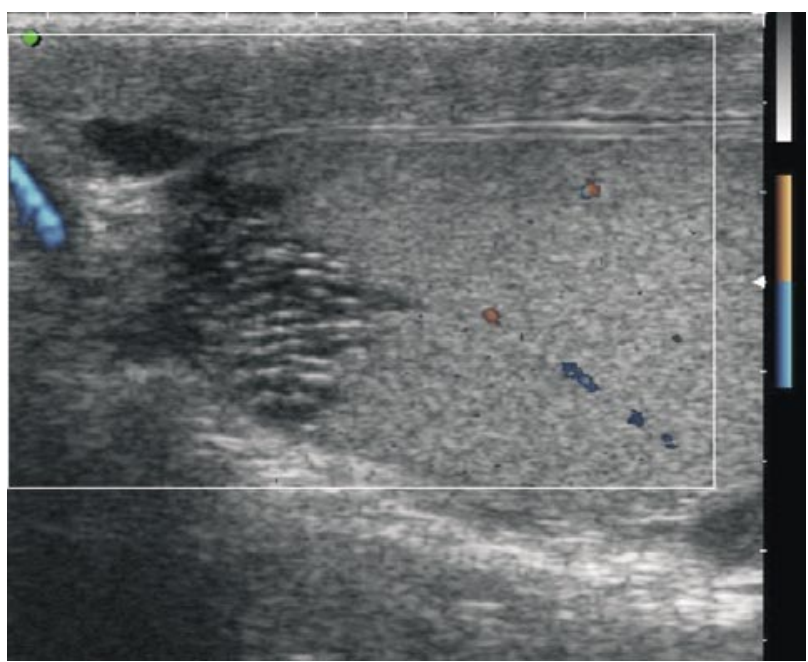

FIGURA 2. Con el eco Doppler color no se observa flujo en estas estructuras quísticas parénquima testicular de mayor ecogenicidad. Aunque el adenocarcinoma de la rete testis es muy poco frecuente se puede distinguir ecográficamente por la presencia de una masa sólida a nivel de la rete testis. Además, la edad del paciente, la forma de presentación clínica y los marcadores séricos tumorales ayudan a hacer el diagnóstico de forma adecuada $(1,2,6,7)$.

Hay que hacer diagnóstico diferencial con otras lesiones benignas intratesticulares, fundamentalmente con la displasia quística de la rete testis y el varicocele intratesticular. La displasia quística es similar ecográficamente e histológicamente, pero se trata de una lesión congénita que afecta a los niños y que se asocia con malformaciones urológicas (8). En el varicocele intratesticular, con el Duplex Doppler existe un patrón venoso de bajo flujo con variación fásica que aumenta con la maniobra de Valsalva (9). Otras lesiones quísticas con las que hay que hacer el dagnóstico diferencial son: quistes de la túnica albugínea, quistes simples testiculares, quiste simple epidermoide y quiste dermoide. Ocasionalmente los hematomas intratesticulares y los abscesos también tienen un componente quístico (10).

Aunque la ecografía es la modalidad de imagen preferida para el estudio del contenido escrotal, también se puede realizar una resonancia magnética nuclear. En las secuencias T2 se ven formaciones multilobulares que son difícil de diferenciar del parénquima testicular normal, mientras que después de la administración de contraste, no realzan y se identifican como formaciones quísticas a nivel del mediastino, estableciendo así el diagnóstico $(8,10)$

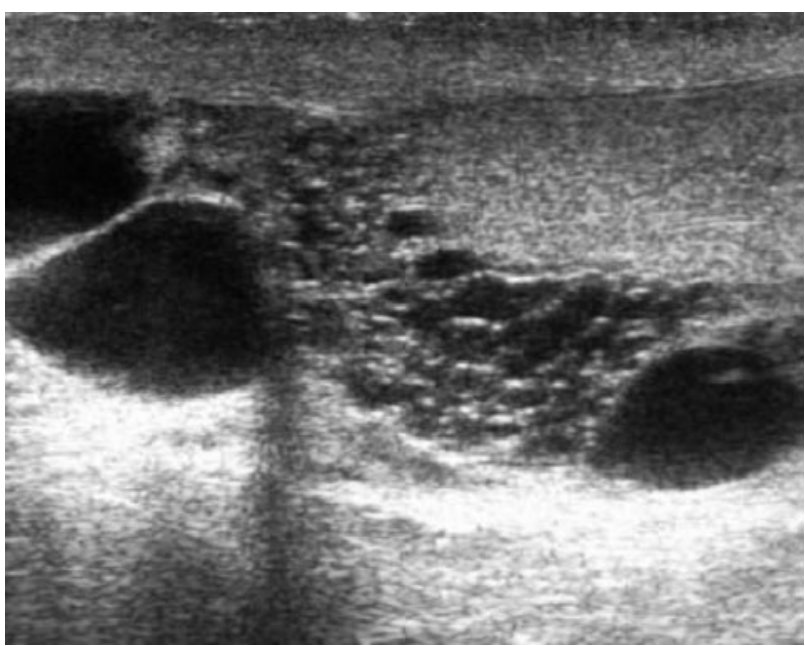

FIGURA 3. En esta imagen se observan quistes epididimarios asociados a la ectasia quística de la rete testis. 


\section{CONCLUSIONES}

El conocimiento de las características ecográficas encontradas en la ectasia quística de la rete testis permite hacer un adecuado diagnóstico de esta entidad benigna sin necesidad de recurrir a la biopsia testicular.

\section{BIBLIOGRAFÍA y LECTURAS RECOMENDADAS ("lectura de interés $y^{* *}$ lectura fundamental)}

*1. HAMM, B.; FOBBE, F.; LOY, V.: "Testicular cysts: differentiation with US and clinical findings". Radiology, 168: 19, 1988.

**2. BROWN, D.L.; BENSON, C.B.; DOHERTY, F.J. y cols.: "Cystic testicular mass caused by dilated rete testis: sonographic findings in 31 cases". AJR Am. J. Roentgenol., 158: 1257, 1992.

3. NISTAL, M.; MATE, A.; PANIAGUA, R.: "Cystic transformation of the rete testis". Am. J. Surg. Pathol., 20: 1231, 1996.

4. CHEHVAL, M.J.; MARTIN, S.A.; ALEXANDER, N.J. y cols.: "The effect of unilateral injury to the vas deferens on the contra-lateral testis in immature and adult rats". J. Urol., 153: 1313, 1995.

*5. BURRUS, J.K.; LOCKHART, M.E.; KENNEY, P.J. y cols.: "Cystic ectasia of the rete testis: clinical and radiographic features". J. Urol., 168: 1436, 2002.

6. DOGRA, V.S.; GOTTLIEB, R.H.; RUBENS, D.J. y cols.: "Testicular epidermoid cysts: sonographic features with histopathologic correlation". J. Clin. Ultrasound., 3: 192, 2001.

7. OLDER, R.A.; WATSON, L.R.: "Tubular ectasia of the rete testis: a benign condition with a sonographic appearance that may be misinterpreted as malignant”. J. Urol., 152: 477, 1994.

8. ROUVIERE, O.; BOUVIER, R.; PANGAUD, C. y cols.: "Tubular ectasia of the rete testis: a potential pitfall in scrotal imaging". Eur. Radiol., 9: $1862,1999$.

9. JIMÉNEZ- LÓPEZ, M.; RAMIREZ-GARRIDO, F.; LÓPEZ-GONZÁLEZ, J. D. y cols.: "Dilatation of the rete testis: ultrasound study". Eur. Radiol., 9: 1327, 1999.

10. MEYER, D.R.; HUPPE, T.; LOCK, U. y cols.: "Pronounced cystic transformation of the Rete testis MRI appearance". Invest Radiol., 34: 600, 1999. 